trained, the disease has excited a lively curiosity as to its nature, and especially as to its mode of transmission. Among the general public there still remains a great fear of the contagiousness of leprosy, evidently issuing from the erronecus interpretation of certain passages of the Old Testament, and the natural repulsion toward the disease. There is an evident desire to get them quickly gone to a remote place with a wish that they may not be overhasty in their return. As a matter of fact, leprosy is very reluctantly contagious, and must require very special favoring circumstances in order for it "to take." A very modest degree of cleanliness hinders its transmission. As soon as a country becomes well to do and adopts the daily tidiness incident to modern civilized life, leprosy dies out. Although, therefore, the Biblical view of the highly contagious nature of leprosy undoubtedly aided in stamping out the epidemic of the middle ages, in modern life it works an unnecessary hardship on the afflicted, and should give place to a more reasonable attitude, agreeing with the real facts in the case.

323 Geary Street.

\section{SYMPTOMS AND DIAGNOSIS OF LEPROSY}

WITH REPCRT OF CASES *

HOWARD MORROW, M.D.

A. W. LEE, M.D.

SAN FRANCISCO

The compilation of notes in this paper is the result of study of the lepers in the Isolation Hospital of San Francisco, of private cases and of observation of patients in the University of California Hospital. The thirteen cases of leprosy in the Isolation Hospital are all chronic, and most of them show evidences of all stages of the disease.

Leprosy is a chronic infectious disease that is produced by a semiacid-fast micro-organism which, morphologically, somewhat resembles the tubercle bacillus. After invasion into the human body this bacillus effects changes in it which are of apparent diagnostic significance.

Leprosy must be regarded as a systemic disease, and may, at some period of its case history, implicate any or all of the units of the human body. This statement can be verified by a direct or indirect manifestation of the malady. By an indirect manifestation of leprosy we mean that, in consequence of the longcontinued loss of bodily resistance, an intercurrent infection is very likely to occur. By a direct manifestation we refer to those instances in which either subjective or objective variations from the normal condition of the body have resulted from the presence of the lepra bacillus in the various organs, as has been proved by laboratory methods of investigation.

As has been stated, while any part of the body may be involved at some epoch in the course of an average leprosy infection, we feel that it is proper to devote most of the detailed division of this paper to those pathologic changes in the integument which may result from it, and particularly are we of this opinion since the great percentage of lepers is recog-

* Read before the Section on Dermatology at the Sixty-Sixth Annual Session of the American Medical Association, San Francisco, June, 1915. nized by skin lesions. These cutaneous evidences of leprosy can be grouped under more or less definite clinical types, but it is quite possible for one variety to merge into another, or to be simultaneously accompanied by every form of integumentary lepra. Evidences of leprosy that appear in the skin may assume the form of a macular eruption, trophic changes following nerve involvement, areas of pigment increase or decrease, and hypertrophic process located in the corium and superimposed structures.

In macular leprosy the lesions may be few or numerous, the affected areas may be large or small, their periphery is usually round or ovoid, their margins as a rule are sharply defined and sometimes elevated. In the beginning these macules are bright red, soon changing to reddish brown. With chronicity they tend to pale in the center, and, by coalescence of their outlines, to form gyrating figures. The margins of such lesions may have a purplish tinge, suggesting a certain form of erythema multiforme of the skin, and at times deep pigmentation occurs in their outermost limits. When central clearing does take place in the macular eruptions of leprosy it assumes a fawn-like color or that of the infected persons' normal integument. Where leprous macules have appeared there is a local decrease of perspiration, a crapelike irregularity of the skin surface and the presence of scales. So far as changes in sensation are concerned, macular leprosy may be entirely free of them during the first few months of its development, or the affected areas may be hyperesthetic. In the later stages, however, these macules are usually anesthetic. When a long-standing macular eruption of leprosy acutely exacerbates, its color flames into a bright red as in its earliest form.

Pigmentary macules are occasionally observed which have not been preceded by any other leprous eruption. And again, areas of depigmentation appear, which, likewise, develop independently of the foregoing manifestations of the disease.

The macular and pigmented varieties of leprosy cause a very inconsiderable interference with the normal functions of the body, and yet in another phase of the infection the pitiable sufferer becomes totally incapacitated for even the simplest employments, and passes into a condition which is as repugnant as that of the most distorted mummy of Egypt or Peru. At this time the nervous system has been directly attacked, and since the ulnar and peroneal nerves are most frequently involved, the greatest deformity results in the hands and feet. Now and then these nerves are so enlarged that they are easily palpable, and in some cases the small cutaneous nerves are so tumefied that they can not only be felt but actually seen. As these pathologic nerve changes go on, contractions occur in the flexor muscles of the hands until the fingers have become so folded on themselves that the condition is commonly termed "the leper claw." The bones of the fingers and toes may be absorbed, in this stage of leprosy, without ulceration, being the result of a rarefying osteitis. This leads to the formation of the so-called "stump" and produces the clinical condition of lepra mutilans. In this variety of nerve leprosy ulcers may be numerous and large, the eyes may be destroyed, the nasal bones absorbed, and the nostrils closed. Fingers and toes are occasionally amputated in a spontaneous manner at this period of the infection. One of the 
rarer manifestations of leprosy associated with the nerve type is the appearance of bullae. These may be seen on any part of the body, but they are generally found on the hands and feet.

Leprosy may announce its presence in other ways from those previously outlined, such as nodular eruptions on the face, limbs and trunk, and clinically this is spoken of as "nodular leprosy." Hoarseness is an early manifestation of the nodular type, due to the presence of lesions in the larynx and pharynx. These submucous nodules have a tendency to ulcerate, but they usually heal during remissions of exacerbations. The lesions can be either large or small, and the face is the part of the body which is as a rule most extensively affected. When the face does show the greater amount of involvement, the nodules are commonly of the large variety; when the body is the site of nodula: formations, the smaller type is the usual finding. Every now and then a case will be observed in which the nodules are not located in the skin proper but occupy a subcutaneous position; in such instances it might be almost impossible to catch the lesions between the examining fingers, as they have formed attachments to the underlying tissues. The nodules of the usual type are frequently elastic to the touch, corresponding to that palpable sensation experienced on manual examination of miliary gummas. It is not unusual to see large comedones in nodular leprosy of the face and trunk. Aside from the distinct lesions in nodular leprosy of the face there is a deep wrinkling of the forehead, a broadening of the nose, a thickening of the lips, a tumefaction of the skin of the outer part of the eyebrows, all of which go to contribute to the well-known "leonine expression" of chronic leprosy. The hair of the eyebrows usually falls out, and that of the scalp becomes dry and tends to a premature graying. In cases of well-marked nodular leprosy the lesions are usually grouped, they are reddish brown, but they assume a brighter hue during exacerbations. They become anesthetic quite early. The nodules might disappear in a few months or persist for years, with or without ulceration. When ulceration does occur healing is followed by cicatrization. When absorption of the nodules takes place without ulceration, areas of cicatricial atrophy or cleep pigmentation mark the location of the vanished lesions.

With or without the presence of active leprosy, particularly in the later stages, a marked desquamation of the skin may occur and usually on the lower limbs; this might be so excessive as to resemble a case of severe ichthyosis.

The subjective disturbances of leprosy are malaise, migratory neuralgic pains, anorexia and depression of both the mental and physical faculties. General hyperhidrosis occurs at times as well as fever, the latter resembling various forms of malaria. All of these symptoms are exaggerated during an exacerbation. Paresthesia and neuralgic pains are associated with the nerve form.

Leprosy of the eyes is such an important suljject that a short description should be given of the usual clinical manifestations. In all types of leprosy the eye is frequently involved and occasionally quite early in the disease.

In the nerve type of leprosy we frequently get excessive lacrimation. This is followed by a marked xerosis causing atrophic shrinking of the mucous membrane and various distortions of the lids. Through paralysis of the lid muscles and inability to close the lids drying of the cornea follows, with exfoliation of the epithelium, deep infiltrates in the parenchyma and involvement of the interior of the globe. Surgical intervention is frequently necessary. In the nodular type of leprosy we find nodules developing in the conjunctiva, in the cornea and in the iris. Iritis is common in the later stages. The deeper nodules in the cornea have a tendency to ulcerate and frequontly we find involvement of the entire uveal tract. This ends in shrunken, painful globes which requires enucleation.

\section{DIAGNOSIS}

Well-advanced cases of nodular leprosy are easily diagnosed. The characteristic leonine expression can seldom be confused with anything else. On the other hand, however, early stages of nodular leprosy noy somewhat resemble syphilis or lupus. Both the large and small forms of nodular leprosy occasionally simulate multiple benign cystic epithelioma and xanthoma tuberosum multiplex.

When only erythematous or pigmentary changes are present a positive diagnosis must be made by the course of the disease and the observation of sensory changes. This is particularly exemplified in the generalized evanescent type which very closely resenubles certain manifestations of erythema multiforme. Only careful investigation of the sensory disturbances will be of value in differentiating ringed leprous lesions from other ringlike cutaneous eruptions; this also applies to the hyperpigmentary and depigmentary forms.

The condition known as lepra mutilans must be distinguished from syringomyelia. In the latter disease there is a dissociation of sensation, and other evidences of leprosy are wanting. In doubtful cases, a portion of the involved nerve may be remover for the purpose of demonstrating the presence of the bacilli; this diagnostic measure has frequently proved to be quite unsatisfactory.

In obtaining material in cases of nodular leprosy to serve the demands of laboratory measures of investigation, a nodule may be excised or a portion of it removed with a biopsy punch. The method which has given us the best service is as follows: The nodule is first incised with a cataract knife and through the opening a small curet is passed deep into the affected area. The material thus removed is smeared over the usual glass slide and stained by the customary acid-fast method. Should the bacilli be present in large numbers, further work is unnecessary; but in those instances in which only a few organisms can be found, cultural procedures and animal inoculation may be resorted to in order to distinguish them from the tubercle bacillus.

In the nodular and mixed type of leprosy, even when syphilis is excluded, the Wassermann reaction is frequently positive. It is positive in almost all cases with leprous antigen. In the anesthetic type it is usually negative, and in those cases in which the reaction is positive it is much weaker thar: in the other forms. In all types of the disease thc lueti: reaction is negative unless the condition is complicated by syphilis.

\section{REPORT OF CASES}

CASE 1.-Jim, aged 34, born in Honolulu, has been in the Isolation Hospital for seven years. He is married, his wife is in good health. His three children all died in infancy. His 
wife's uncle had leprosy. He came to California from the Hawaiian Islands nine years ago, and at that time had one perforating ulcer on the sole. Nodules developed soon after. He now has an unusually severe type of nodular lepra of the face, body and limbs. No hair appears on the face; the hair on his scalp is thick, dry and prematurely gray. Leprous nodules are on the hard palate. On the body the eruption consists of grouped lenticular papules, a few tuberous growths and some ulcers. The ulcers are most numerous on the hands, where they are the result of burns. There is atrophy of the testes. Atrophy of the limbs is beginning and anesthesia of the limbs is progressing. There are distinct leprous nodules in the left palm and many pigmented spots. Many perforating ulcers are on the feet. The right eye shows infiltration and edema of the temporal side of the ocular conjunctiva with a small patch of whitish infiltration of the contiguous portion of the cornea. There is no involvement of the pupillary area of cornea and the sight is good. The left eye shows similar involvement of the ocular conjunctiva, most marked at the lower temporal border of the limbus. The cornea is clear and sight is good. Wassermann negative with syphilitic antigen and positive with leprous antigen.

Case 2.-James, a colored man, aged 63 , was born in Maryland. He went as cook with the Army to the Philippines and returned to California in 1899 . The eruption appeared in 1908 as miliary nodules over his face. Late: nodules developed in the eyes. There is still hair on brows and lids. The nodules on the face have been absorbed. His body is clear. The skin on the backs of his hands is shiny, thickened and there is much of the usual tumefaction with some scattered nodules. There is some muscular atrophy, but sensation is still good. The skin of the legs is dry, shiny, scaly and darker than the rest of the body. His right eye was entucleated three years ago to relieve the pain which resulted from the presence of leprous nodules in the cornea. His left eye shows nodular thickening on the center of the upper lid. There is a grayish white ring of opacity surrounding the cornea which is broadest at the superior aspect and is similar to an arcus senilis. Punctate gray opaque spots appear througl the center of the cornea. The sclera shows the irregular pigment of the African races. Wassermann negative with syphilitic antigen; positive with leprous antigen.

Case 3.-Fong Gong, a Chinese, aged 47, shows the nodular type. On the face the nodules are very large and are most numerous on chin, cheeks and brows. He has the saddle nose and leonine expression. The hair on his scalp is normal, but has disappeared from his brows and lids. $\mathrm{His}$ ears are enlarged and anesthetic. Many large comedones are in the nodules on the nose and cheeks. His hands show much tumefaction, with the characteristic dark brown skit with a bluish tinge. His palms show the characteristic dryness. There is partial anesthesia of the hands, but muscular atrophy is not marked. The skin of his trunk, particularly of the back, shows a diffuse thickening, with exaggeration of the normal marh.ngs and a diffuse confluent macula: fawn-colored eruption. Scattered nodules of a deeper brown hue can be seen over the affected area. This condition was much more marked a few years ago and is an excellent example of elephant skin. Anesthetic areas are scattered over the body and some of the nodules are anesthetic. There is a diffuse thickening of the skin of the legs, with dark brownish discoloration and wrinkling, and a nodular cruption on the sole. Complete anesthesia of the feet exists. A perforating ulcer is on the ball of the right big toe. Nodules on the right eyelids are so large as to mechanically hinde: their opening. The right eye shows quadrilateral atrophy of the globe. There is applantation of the cornea with numerous grayish cicatrices. Even light perception is gone. The left eye shows grayish white infiltrates of the cornea in various stages of activity. There is photophobia. Partial sight remains.

CASE 4.-Bill, aged 34, a sailor, born in Honolulu of English parents, has been in the Isolation Hospital for three ycars. The trouble started four years ago as a thickening of the nose and ears. The skin on his face is crapelike and yellowish gray. The normal markings are exaggerated. There is xanthelasma and right facial palsy, with a constant rhythmical contraction of the muscles about the right eye. The lids are normal except for the right palsy. The conjunctiva of the globes is injected. The hair in the brows is greatiy diminished. The ears are enlarged and the skin over them is shriveled. Sensation on the face is delayed and decreased. There is also decreased sensation on the forearms and hands, mostly in distribution of the ulnar nerves. Thenar and hypothenar eminences are atrophied. The palms are dry and the normal markings accentuated. The fingers are thickened and clubbed and the skin is shiny. The color here is browner than that of the rest of the body. The skin of the feet is brown and shiny and the soles are deeply pigmented in places. There are no sensory changes. On the forearms and legs are a dozen subcutaneous nodules, rather poorly defined, the skin over them being a bit elevated, and over some there is slight redness. These last a few weeks, disappear, and are then followed by others. These are anesthetic, and leprous bacilli are present in them.

CASE 5.-Dan, aged 30, born in Greece, came to America thirteen years ago and has been in the Isolation Hospital fo: four years. Symptoms were first noticed in 1910 as lesions in the eyes. Later he had papules on cheeks and thighs, and excision of one of these papules showed numerous acidfast bacilli. Later he developed muscular atrophy of the hands. He now has lepra nodules sparingly distributed over his face. The hair has fallen from the cheeks and entirely from the brows. Nodules appear on the soft and hard palate. There are a few nodules on the backs of his hands on a skin which is reddish brown, dry, shiny and crapelike. His palms are dry. Atrophy of the thenar and hypothenar eminences exists, but there is still much muscular power and sensation in the hands is still good. The center of the cornea in the right eye is the site of an ectatic scar with many long blood vessels from the surrounding sclera. The anterior chamber is deep. The pupil is irregular from an old iritis. The left eye is similar to the right. He counts fingers with the right eye and light perception with the left. Wassermann was negative with syphilitic antigen and positive with leprous antigen.

CASE 6.-Ah Fong, aged 33, born in Canton, has been at the Isolation Hospital for four years. Dull reddish-brown macules appear on face. These are fading. Areas of dark brown pigmentation are on the thighs; the skin of thighs, legs and feet is scaly and dark brown. Sensation is diminished, especially on the lower limbs.

CASE 7.-Yee Foo, aged 57, has been in the Isolation Hospital for twelve years. For five years before his entrance the patient had an eruption on his face of the nodular type of leprosy. The nodules have been large and numerous on the face, but nearly all have resoluted, leaving a dark brown pigmentation, especially on the cheeks. The hair is still in brows and lids; the ears are normal. No marked change in sensation exists on the face. It is many years since these nodules have shown active signs. The skin on the body is dry, wrinkled, shiny and with little change in color. The palms are dry. There is some contraction of the little fingers and some muscular atrophy. There is also delayed and decreased sensation. These changes in the hands are recent, showing the condition to be active in the nerves and thus producing a mixed type of leprosy. The right eye is negative except for a few old scars. The left eye shows injection of sciera, most marked above and below. A grayish-white infiltrate of the cornea extends $4 \mathrm{~mm}$. from the upper limbus. The lower portion of the cornea shows a few whitish spots of infiltrate. The iris is thickened and discolored; sight is diminished.

CASE 8.-Frank, a Chinese, born in Canton, aged 32, has becn in the Isolation Hospital for one and one-half years. The trouble began three years ago with loss of feeling in the hands; then macules appeared on the face. Since that time these have cleared. Some areas of anesthesia are on his face and remains of an indistinct macular eruption appear 
on the lower part of his back. These areas are hyperesthetic. A fading erythema is on the thighs and legs. These lesions are indistinct, browner than the normal skin, a bit crapelike and very dry. There is decreased and delayed sensation. The ulnar nerves are enlarged. The fingers are contracted and clubbed; the palms are dry and the thenar eminences are atrophied. The interossei are markedly atrophied. The hands are anesthetic, eyes negative. No lesions appear in his mouth.

Case 9.-Pedro, aged 40, born in Alcapulco, Mexico, has been in the Isolation Hospital for twenty-five years. Botl ocular globes were enucleated three years ago. The right lower lid shows marked ectropion; the upper lid is normal. The conjunctiva of the lids is smooth, dark red and without nodules; the left lids are similar. There is short, sharp, nonrhythmical contraction of the muscles about the mouth, and shriveling of the skin over both cheeks, aboui the angles of the mouth and of the chin. The patient has claw hands and the muscles are markedly atrophied, but there is very little change in the nails. The legs are atrophied. The skin is scaly and dark reddish brown. The patient has foot-drop from paralysis of the peroneal and tibialis anticus. The toenails are somewhat atrophied.

CASE 10.-Lem Him, aged 50, born near Canton, has been in the Isolation Hospital for eight years. The disease is of the nerve type. His face looks normal, but the skin is almost completely anesthetic. The brows are normal. There are pigmented spots over the trunk. His hands show marked muscular atrophy and the skin is completely anesthetic. Many digits have been absorbed. Three contractured fingers remain on the right hand. All phalanges on the left hand are more or less absorbed. The skin is wrinkled on the back of the hands and somewhat reddened. The ulnar nerves are enlarged. The legs are atrophied and the skin in places is pigmented. The right foot is clubbed. The feet and legs are anesthetic. The testes, are atrophied. The right eye shows an arcus senilis and a small pterygium from the nasal side.

CASE 11.-How King, aged 54 , born in Canton, has lepra mutilans. As an example of the mummifying type of the lepra, the following case serves as an example. Both eyes are blind from atrophy of globes. The nose is flat from destruction of the cartilaginous and bony structures and partial closure of the nostrils. The skin of the body is brown and wrinkled. The thighs and legs are very dark and scales are large, the condition simulating a scvere type of ichthyosis. The hands are badly deformed, the fingers contracted into the palms and wrists flexed. There is very little absorption of the phalanges. The fingernails are atrophied. The taes are contracted and fect are clubbed. Some of the toenails are hypertrophied, some are atrophicu and some are absent. This man is helpless, has been confined to his room for many months, and yet seems no nearer death than a year ago.

CASE 12.-P. P., an old railroad employee, aged 40, born in Greece, has been in America for thirteen years and in the Isolation Hospital for seven years. This is an example of mixed leprosy. Large flat pale nodules are scattered over the face, particularly on the forehead. This man is blind. The palpebral apertures on the right side are smaller than on the left. The skin of the lids is thickened and pale and cilia are missing in places. No hair is in the brows. There is a quick, jerky, horizontal nystagmus. The globes show quadrilateral atrophy with applantation of the corneas, which are opaque, and the anterior chambers are abolished. Light perception is present. The skin of the entire body is dry and a bit scaly. There are nodules and resoluted nodules on the forearms as well as scars from old ulcers. The hands show the nerve type of the disease, with atrophy of muscles and contractured little fingers. The hands and forearms are anesthetic and tactile sensation is gone. The nodules on the face are not anesthetic.

CAsE 13.-Mike, aged 34, born in Greece, has been in America for six years and in the Isolation Hospital for two and one-half years. He gives a history of fever for a year. Then he noticed an eruption of the nodular type on his forehead. Large, bright red nodules are on the face, especially on the forehead and cheeks. No lesions appear on the nose and some hair is still present in the brows There is no change in sensation of skin of face. This man has had several exacerbations, and during the attacks the nodules are much larger and the color is much brighter. Dark reddish-brown nodules appear on the forearms, wrists and backs of the hands; also atrophic scars and stains from absorbed nodules. There is no muscular atrophy in the hands, but there is delayed sensation. The ulnar nerves feel quite large. On the body nodules are scattered over the back. On the anterior surfaces of the thighs there are scattered pigmented and atrophic areas. Over the buttocks and posterior surfaces of the thighs are grouped nodules and deep stains. On the legs, particularly the lower third, are large and small nodules and a few small nodules appear on the soles. The larger nodules are anesthetic. The right eye shows at the temporal side of the cornea a few injected blood vessels. The left eye shows edema of the cornea, pericorneal injection and a pinguecula at the nasal side. There is some pain in the eyes and photophobia. The edema of the cornea is an early symptom of subsequent ulceration.

135 Stockton Street-1107 Stanyan Street.

\section{THE ETIOLOGY AND TREATMENT OF LEPROSY *}

\section{ERNEST DWIGHT CHIPMAN, M.D. SAN FRANCISCO}

It may be granted to be axiomatic that the more complete our knowledge of the cause of a disease the more perfect our prophylaxis and the more rational our treatment. The more we have to deal with conjecture concerning etiology the more uncertain our efforts at control and the more empiric our treatment.

A review of recent progress in the treatment of leprosy necessarily goes hand in hand with a recapitulation of advances made in the search for data relating to its propagation and transmission.

While the lepra bacillus of Hansen is generally conceded to be the specific cause of the disease, its route of entry is unknown, and many important questions concerning contagion remain unsettled.

The theory of direct hereditary transmission is practically disproved.

The precise manner in which leprosy is acquired is as yet unsettled. The theory that the disease is caused by the eating of decayed fish apparently has fewer and fewer adherents, although prior to the successful cultivation of the bacillus it was argued that the bacillus could not lead a saprophytic existence because no medium could be found on which it would grow.

That the disease is spread by direct inoculation would seem improbable from the uniformly negative results in numerous human and animal experiments.

The part played by insects in the dissemination of leprosy has received much attention of late.

Currie, working in the Hawaiian Islands, concluded that mosquitoes feeding under natural conditions on patients with nodular leprosy so rarely, if ever, imbibe the lepra bacillus that we can exclude them as one of the ordinary means of transference of this bacillus from lepers to the skin of healthy persons. The reason for this is that the mosquito in biting goes directly

* Read before the Section on Dermatology at the Sixty-Sixtl Annual Session of the American Medical Association, San Francisco, June, 1915. 